\title{
Correction to: Heterogeneity in social values and capital accumulation in a changing world
}

\author{
Pierre Gosselin ${ }^{1}$ • Aïleen Lotz ${ }^{2}$ - Marc Wambst ${ }^{3}$
}

Published online: 21 April 2018

(C) Springer-Verlag GmbH Germany, part of Springer Nature 2018

\section{Correction to: J Econ Interact Coord https://doi.org/10.1007/s11403-018-0220-0}

In the printed version of the article, the conditions for the derivatives of Eq. (5) in Sect. 3.2 contained several misprints. The correct version reads as follows:

$$
Y_{t}=\eta\left(\phi_{t} \cdot \chi_{t}\right)\left(\xi K_{2}\left(\xi, \eta, \phi_{t} . V_{t}\right) V_{t}+(1-\xi) K_{1}\left(\xi, \eta, \phi_{t} \cdot \chi_{t}\right) \chi_{t}\right)-\eta\left(\phi_{t}\right)
$$

So that the derivatives satisfy the following conditions: $\left(\xi K_{2}\right)_{1}>0,\left((1-\xi) K_{1}\right)_{1}<$ $0,\left(\xi K_{2}\right)_{1,1}<0,\left((1-\xi) K_{1}\right)_{1,1}<0,\left(K_{i}\right)_{3}>0,\left(K_{i}\right)_{3,3}<0$ for $i=1,2$."

The original article can be found online at https://doi.org/10.1007/s11403-018-0220-0.

$凶$ Pierre Gosselin

Pierre.Gosselin@univ-grenoble-alpes.fr

Aileen Lotz

a.lotz@erc-cercatrova.eu

Marc Wambst

wambst@math.unistra.fr

1 Institut Fourier, UMR 5582 CNRS-UJF, Université Grenoble Alpes, BP 74, 38402 St Martin d'Héres, France

2 Cerca Trova, BP 114, 38001 Grenoble Cedex 1, France

3 IRMA, UMR 7501, CNRS, Université de Strasbourg, 7, rue René Descartes, 67084 Strasbourg Cedex, France 